\title{
Antibiofilm and Antioxidant Activity of Formulated Nanoemulsion from Ocimum tenuiflorum ${ }^{\dagger}$
}

\author{
Ponnarmadha S. ${ }^{1, *}$, Vasanth K. ${ }^{1}$, Vijayanand S. ${ }^{1}$ \\ 1 Department of Biotechnology, Bannari Amman Institute of Technology, Sathyamangalam, Erode-638401 \\ * Correspondence: ponnarmadha@bitsathy.ac.in; \\ $\dagger$ Presented at International Conference on Bioengineering for Health and Environment (ICBHE 2020)
}

Received: 5.07.2020; Revised: 10.07.2020; Accepted: 12.07.2020; Published: 15.07.2020

\begin{abstract}
Nanoemulsions are nano-sized emulsions, which are made for improving the delivery of active pharmaceutical ingredients. These are the thermodynamically steady isotropic system where two immiscible liquids are mixed to form a single-phase by means of an emulsifying agent, i.e., surfactant and co-surfactant. In this study, the extracts of essential oils from medicinal plants, namely, Ocimum tenuiflorum (Tulasi) is used, which have antimicrobial, antioxidant, antibiofilm, and anti-inflammatory activities. Oil in water emulsions was prepared by high-pressure homogenizer using plant essential oils with water as a dispersion medium. The physical and mechanical stability tests were carried out. The emulsions were subjected to antibiofilm assays, and results were compared to estimate the anti-biofilm efficacy of the emulsions. The emulsions were prepared by varying concentrations and time to determine the particle size distribution. These results proposed for developed plant essential oil-based nanoemulsion is thermodynamically stable, and formulated nanoemulsion will be used in various biomedical applications, textile industry applications, and food industry.
\end{abstract}

Keywords: Nanoemulsion; Homogenizer; Surfactant; Antibiofilm activity; Oil in water emulsion.

(C) 2020 by the authors. This article is an open-access article distributed under the terms and conditions of the Creative Commons Attribution (CC BY) license (https://creativecommons.org/licenses/by/4.0/).

\section{Funding}

This research received no external funding.

\section{Acknowledgments}

This research has no acknowledgment.

\section{Conflicts of Interest}

The authors declare no conflict of interest. 